\title{
Lifetime Maximization on Scalable Stable Election Protocol for Large Scale Traffic Engineering
}

\author{
Muhammad Asad ${ }^{\star}$, Arsalan Ali Shaikh, Soomro Pir Dino, Muhammad Aslam and Yao Nianmin \\ School of Computer Science and Technology, Dalian University of Technology, Dalian, China
}

\begin{abstract}
Recently, Wireless Sensor Networks (WSNs) are getting more fame because of low cost and easy to manage and maintain. WSNs consists of sensor nodes and a Base Station (BS). Sensor nodes are responsible to sense, transmit and receive the data packets from sensing field, and the BS is responsible to collect this data and covert it into readable form. The main issue in this network is lack of power resources. As sensor nodes are restricted to limited energy, so researchers always aims to produce an energy efficient clustered routing protocol. To make the efficient routing protocol, heterogeneity of sensor nodes is a best possible solution. 'Stable Election Protocol' was the first heterogeneous network and proposed two level of heterogeneity. SEP Protocol not only improved the network lifetime but also improved the stability of sensor nodes. In order to maximize the network lifetime, we propose the scalability of SEP routing protocol (S-SEP) to check the reliability in large scale networks for traffic engineering. We compare the results of standard SEP routing protocol with fourth level of heterogeneity. Simulation results proves that S-SEP protocol works more better in larger networks.
\end{abstract}

Keywords-Wireless sensor networks (WSN); heterogeneous network; clustered routing protocol; traffic engineering

\section{INTRODUCTION}

Wireless Sensor Networks (WSNs) is the unique network consists of small size sensor nodes which contains battery for energy, radio for transmission and reception of data packets, processor for processing on data packets and storage to store the data packets [1]-[3]. These sensor nodes are responsible to sense the sensing field. When the event occurs these sensor nodes sense this event and collect the data. After collection of this data these sensor nodes transmit data packet towards Base Station (BS). As these sensor nodes are limited to energy resources and their embedded batteries are not replaceable once they have deployed in the network, so the design of routing protocol has to be energy efficient in order to maximize the network lifetime [4], [5]. In the past, conventional routing protocols use two featured techniques for data transmission, Direct Transmission (DT) and Minimum Transmission Energy (MTE). In the DT sensor nodes transmit sensed data directly to the BS which results the early death of nodes which are far from BS because of huge amount of energy dissipation due to long range transmission while in MTE, data packets are delivered through routes. Nodes which are supposed to transmit data, forwards the data packets to the nodes which locates near to the BS which results the early death of relay nodes because of huge amount of data transmission [6]-[8]. Both transmission techniques does not suitable for longer network lifetime. LEACH proposed a solution by dividing the network field into clusters and choose a random Cluster Head $(\mathrm{CH})$ in each cluster for a specific round which is responsible for data transmission of all the nodes locates in its cluster [9]. $\mathrm{CH}$ is chosen dynamically through proper $\mathrm{CH}$ selection and each node have a equal chance to become $\mathrm{CH}$. This random distribution of energy resources among all the nodes control the network load and produce a better network lifetime. But LEACH was a homogeneous network and all the nodes in the network have the same amount of energy which results the unreliability in the large scale of network. To complete the requirement of some applications of maximum lifetime, SEP was proposed with the heterogeneous network [10]. SEP proposed two level of heterogeneity in which two types of nodes was deployed in the network. First one was normal nodes and the others was advance nodes. Advance nodes have some extra energy resources more than normal nodes and $\mathrm{CH}$ selection was purely based on residual energy of sensor nodes. This scheme results in longer lifetime because BS always choose the node as $\mathrm{CH}$ which has more energy and rest of the nodes act as member nodes in a specific cluster. Simulation results of SEP routing protocol proved that SEP produce better network lifetime with more stability in the network. But due to the two level heterogeneity SEP does not work for some specific applications. There are some application which require the huge density of sensor nodes in a very large scale network and these application require longer network lifetime due to the cost of sensor nodes. In this regard we proposed Scalability of SEP routing protocol which is enough adaptive to accept new nodes deployed at any time in the network. Our contribution in this paper is to deploy sensor nodes with different level of heterogeneity in different dimensions of network. In order to check the reliability of protocol, we proposes the fourth level of heterogeneity and deployed the SEP routing protocol in four different scenarios. Simulation results proves that scalable SEP extends the stability period and network lifetime in large scale network.

The rest of the paper is organized as follows: Problem definition and detailed explanation of routing protocol is given Section II. Simulation results are discussed in Section III and we conclude this paper in Section IV.

\section{Scalable Stable Election Routing Protocol}

In this section, we briefly explain the potentials of SEP routing protocol and why it is necessary to be deployed in large scale of networks. This section contains the problem definition and network models which are explained in the subsections.

\section{A. Problem Definition}

As WSNs are emerging from the past decades, the requirements of applications are also getting high with technologies. When SEP routing protocol was proposed, it was easy to 
manage due to small scale of applications. But recently, applications like, military surveillance, vehicle monitoring, environmental control, harvest monitoring, etc are getting vast [11]. So the previous protocol is difficult to deploy in such application due to its limited network lifetime. Nowadays, applications requires maximum network lifetime so it work for a longer period. Furthermore, these application are not limited to lifetime of network, they also require the adaptability of network. Because in the past, when the network is completely dead they need to deploy a complete new network. But due to the recent technologies, sensor nodes are enough intelligent to adopt the network at any time [12]. In this regard, we further enhance the scalability of SEP routing protocol to adopt these sensor nodes through $\mathrm{CH}$ selection. When the network is about to dead, these intelligent nodes can be added in the network and the whole network will become normal again. Through this addition of sensor nodes, scalability issue is resolved which is proved in simulation results.

\section{B. Network Topology}

In the network model of scalable SEP routing protocol, initially 100 static nodes are deployed in the network of $100 m \times 100 m$ sensing field while the energy-free BS is located in the center of the network. All the dimensions of the network is known and BS knows the location of each sensor nodes. As we described earlier that SEP is completely distributed heterogeneous-aware routing protocol, so the $\mathrm{CH}$ selection is based on initial energy of sensor nodes for the first round. Heterogeneous nodes are deployed in the network with the initial energy $1 j$ so the probability of being a $\mathrm{CH}$ is always between advanced nodes. After finishing of first round, $\mathrm{CH}$ selection will based upon remaining energy of sensor nodes. Percentage of advance nodes is $30 \%$ and the normal nodes are $70 \%$ deployed in the network. Fig. 1 shows the network topology of S-SEP routing protocol, in which normal nodes, advance nodes and BS can be seen clearly while Fig. 2 shows the clustered topology of S-SEP routing protocol.

\section{Heterogeneous Network Model}

As we describe earlier, SEP routing protocol is purely heterogeneous network which deploy network in distributed manner. Heterogeneous networks in WSNs are solely proposed to improved the network lifetime. Initially, there were only single type of nodes which has same energy levels. But heterogeneous network provide different levels of energies to sensor nodes which results in better network lifetime [13]. Two level of heterogeneous nodes are deployed in network, one is called as normal nodes and the other is advanced nodes. Normal nodes have the initial of $0.5 j$ while the advanced nodes have initial energy of $1 j$. This two level of heterogeneity was proposed by SEP routing protocol. In this paper we took heterogeneity to the fourth level with the initial energy of advanced nodes to $1.5 j$ and $2 j$, respectively.

\section{Radio Model}

Sensor nodes depicts the energy in sensing, data collecting, data processing and data transmission but the energy consumed in communication is greater than other dissipation of energies, so the communication energy cannot be negligible. That is why researchers always focus to minimize energy consumed in transmission process of routing protocols. As we mentioned in the first section that sensor nodes consist of radio and this radio consist of transmitter and receiver shown in Fig. 3. For the fair comparison of lifetime enhancement, we consider first order radio model and free space model which was adopted in previous routing protocols [14]. Proposed scalable SEP routing protocol dissipates $50 \mathrm{nj} / \mathrm{bit}$ of energy for transmission and reception of data packet. In order to transmit the data packet $(d p)$ at the distance $d$ the total transmitted energy can be calculated as:

$$
T_{T E}(d p, d)= \begin{cases}d p \times E_{\text {elec }}+E_{f s} d^{2} \times d p, & \text { if } d<d o \\ d p \times E_{\text {elec }}+E_{a m p} d^{4} \times d p, & \text { if } d \geq d o .\end{cases}
$$

Where, $T_{T E}(d p, d)$ is the total transmitted energy and $E_{f s} d^{2} E_{a m p} d^{4}$ represents the free space and first order radio models respectively. While, $d p$ represents the data packet and $d$ is the distance between source to destination.

\section{E. Cluster-Head Selection Model}

In this section, random $\mathrm{CH}$ selection is explained [15]. Algorithm performs the intelligent selection of $\mathrm{CH}$ based on residual energy of sensor nodes. First, routing protocol establish the network topology and distribute the nodes equally among clusters to balance the load. After that protocol gives the authority to BS to select the suitable CHs. As described in the network model, two types of nodes are deployed in the network; normal nodes recognized as a $N_{n}$ and advance nodes as $N_{a}$. BS calculates the suitability of sensor nodes in order to select the $\mathrm{CH}$. To calculate the suitability of sensor nodes, BS must be familiar with these properties of sensor nodes: initial energy, residual energy, energy consumption ratio $(E C R)$ and distance of node to BS. So, in order to calculate the suitability BS needs to calculate the energy consumption ratio of each node in the network. Following equation is used to calculate the $E C R$ :

$$
E C R_{N}=\frac{E_{o}}{E_{o}-E_{r}}
$$

Where, $\mathrm{N}$ denotes the total number of sensor nodes in the network, $E_{o}$ is the initial energy and $E_{r}$ is the remaining energy of sensor nodes. After getting familiar with $E C R$ BS will be able to calculate the suitability of each sensor node.

$$
\text { Suitability }_{N}=\frac{E_{r}}{E C R \times d}
$$

Where, $d$ is the distance between sensor nodes and BS. After calculating the suitability, BS selects the desired percentage $P$ of cluster heads. As SEP routing protocol use the epoch $N_{i}=1 / P_{\text {optimum }}$ to select $\mathrm{CH}$ so after each round new $\mathrm{CH}$ will be selected based on residual energy.

When all the $\mathrm{CHs}$ are selected, hello packets will be exchanged between all the sensor nodes. At the initialization stage of network, BS transmits the hello packet to all the sensor nodes and the packet contains the node ID, cluster ID, $\mathrm{CH}$ and 


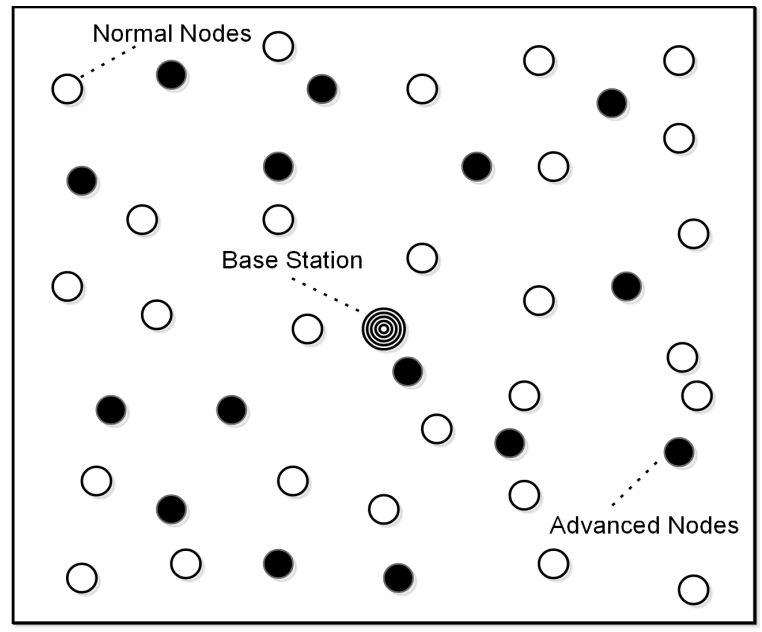

Fig. 1. Network model.

\section{$\mathrm{H}$}

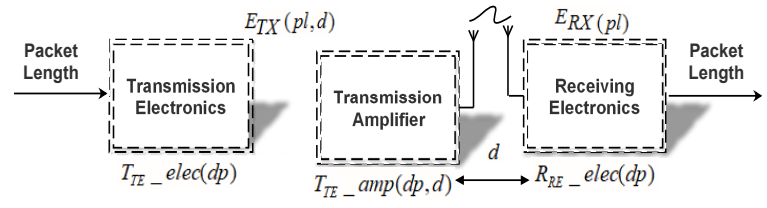

Fig. 3. Radio model.

total network energy. In order to calculate the total network energy following equation is used:

$$
\operatorname{Total}_{\text {Energy }}=\sum_{i=1}^{N_{i}} E_{o}(1+\alpha)
$$

Average energy of the network will be calculated in the similar fashion:

$$
\text { Average }_{\text {Energy }}=\frac{1}{N_{i}} \sum_{i=1}^{E_{i}} E_{i}\left(A_{E}\right)
$$

In order to select the appropriate number of $\mathrm{CH}$ for the particular nodes, the equation applies:

$$
P_{C H}=P_{\text {optimal }} \frac{E_{i}}{E_{i_{A E}}}
$$

$P_{\text {optimal }}$ is the percentage to become $\mathrm{CH}$, initially all nodes have same percentage to become $\mathrm{CH}$. As the routing protocol is rotating epoch, so the nodes become $\mathrm{CH}$ in first round they won't be able to become $\mathrm{CH}$ in the next round because selection of protocol is based on residual energy of sensor nodes, Fig. 4 and 5 shows the selection of $\mathrm{CH}$ with respect to rounds. In order to maintain the same probability of $\mathrm{CHs}$ in each round, BS choose a random number between [0-1]. If random number is less than value of threshold then the node become $\mathrm{CH}$ for the current round otherwise it will be selected as a member node of specific cluster. To calculate the value of threshold, equation applies:

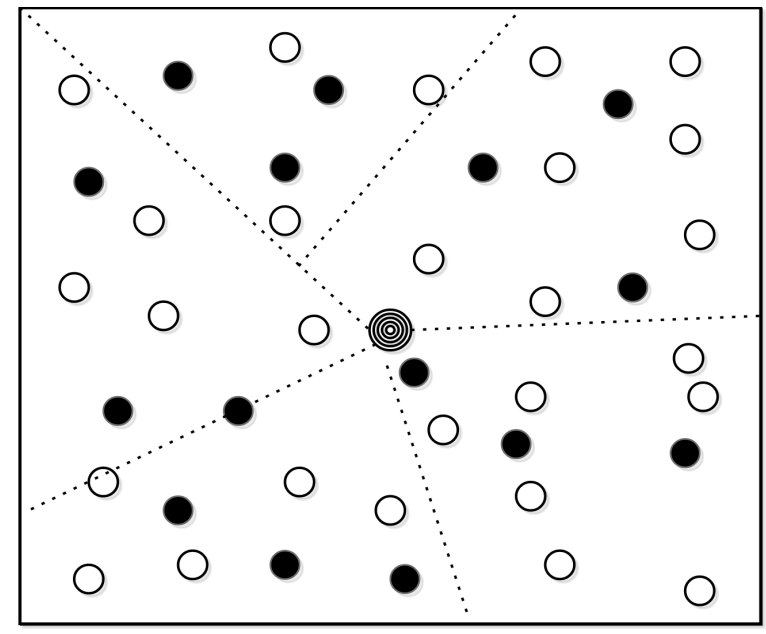

Fig. 2. Clustered.

$$
\text { Threshold }= \begin{cases}\frac{P_{\text {optimal }}}{1-P_{\text {optimal }} \times\left(\text { rmod } \frac{1}{P_{\text {optimal }}}\right)}, & \text { if } N \in G . \\ 0, & \text { otherwise }\end{cases}
$$

where, $r$ is the running round, $N$ are the nodes and $G$ is the set of $\mathrm{CHs}$ and $d$ is the distance between node to BS. In order to calculate the desired percentage of $\mathrm{CH}$ between normal nodes and advance nodes according to their energy levels, the formulation applies:

$$
\begin{gathered}
P_{\text {Normal }}=\frac{P_{\text {optimal }}}{1+\alpha \times N} \\
P_{\text {Advanced }}=\frac{P_{\text {optimal }}(1+\alpha \times N)}{1+\alpha \times N}
\end{gathered}
$$

Equations shows the extra heterogeneous energy in advance nodes. The above two equations shows the two level heterogeneity in standard SEP routing protocol. But in order to add more heterogeneity in the network to make it scalable for large scale, the model applies:

$$
P_{S}=\frac{P_{\text {optimal }} \times N(1+\alpha)}{N+\sum_{i=1}^{N} \alpha}
$$

where $P_{S}$ shows the scalability of routing protocol, equation proves that protocol is reliable for large scale network and it is adoptable to add more nodes with different level of heterogeneity in the network.

Above all equation shows the proposed scalability of SEP routing protocol. Models prove that multiple number of heterogeneity can be added in the network to improve the network lifetime and this addition of multiple heterogeneity does not interrupt the $\mathrm{CH}$ selection. It is proved that newly added nodes have more chances to become $\mathrm{CH}$ due to higher energy and ultimately this feature will enhance the network stability and network lifetime in real-time applications of present day. 


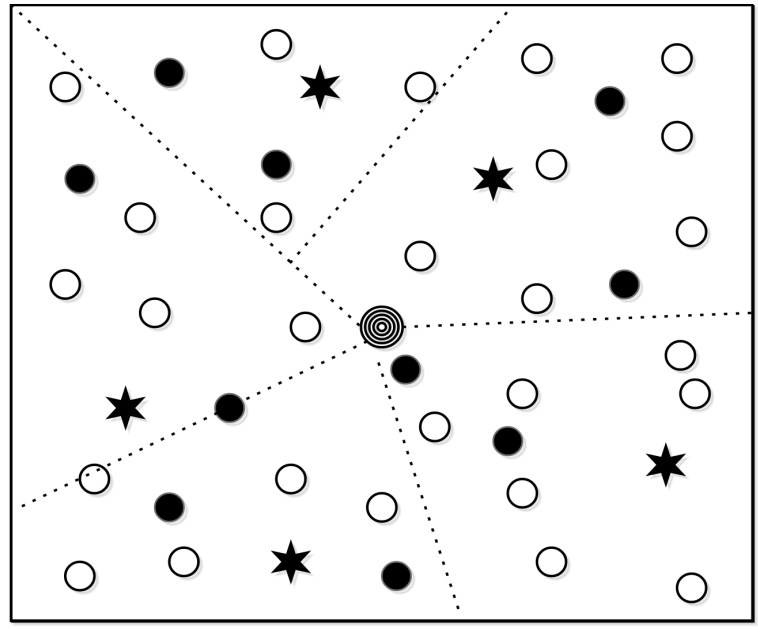

Fig. 4. Selected cluster-heads for running round.

\section{F. Association of Member Nodes to CHs}

When all the $\mathrm{CHs}$ are chosen, then the association of member nodes with their perspective $\mathrm{CH}$ will begin. $\mathrm{CH}$ runs the CSMA MAC protocol and sends the hello packet to all neighbour nodes with the flag of $\mathrm{CH}$. All neighbouring nodes will receive this message and response with their location and ID. All the member nodes will remain active during this communication between nodes and $\mathrm{CHs}$. When $\mathrm{CHs}$ receive the location and IDs of member nodes it will associate these nodes with itself. In order to calculate the range of $\mathrm{CH}$ for associating nodes, the equation applies:

$$
\text { Nodes }_{\text {association }}=\frac{R S S I}{d_{C H}}
$$

where, RSSI denoted the received signal strength indication $d_{C H}$ shows the distance of $\mathrm{CH}$ to member nodes. After the association with $\mathrm{CHs}$, member nodes will receive the TDMA slots from CHs. These slots are allocated to nodes to avoid interruption between communication of member nodes and CHs. Each node will only communicate with $\mathrm{CH}$ in its own allocated slot. Only during this time period nodes activate their transmitter to transmit their sensed data to $\mathrm{CHs}$ and the rest of the time transmitter will be in sleep mode. This extra property of energy saving will further enhance the network lifetime.

\section{G. Transmission of Data Packets to BS}

After the association of member nodes with their prospective $\mathrm{CHs}$, nodes transmit their sensed data to $\mathrm{CH}$ in their allocated time slot. During the whole network time $\mathrm{CH}$ will remain active to receive data from sensor nodes and perform the responsibility as a $\mathrm{CH}$. $\mathrm{CHs}$ will compress all the data packets using multiple signal processing techniques and aggregate the meaning full data to the BS.

\section{RESULTS DisCUSSION}

In this section, we simulate Stable Election Protocol (SEP) in multiple networks with multiple network dimensions. First we simulate SEP routing protocol in MATLAB on different heterogeneity levels and then gather the data in Origin9.1 to

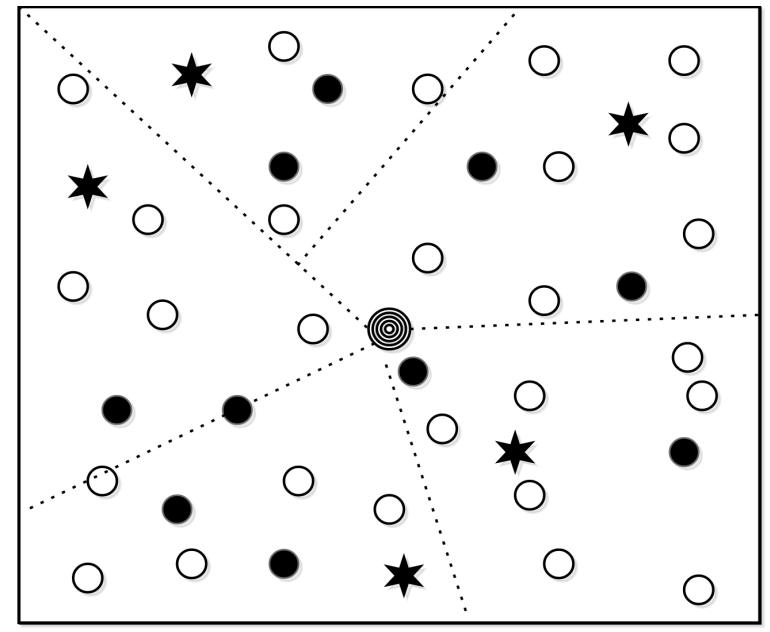

Fig. 5. Selected cluster-heads for running round $\mathrm{R}++$.

produce the results. In this paper, we check the scalability of SEP routing protocol for large scale network. In order to prove the reliability we took four different scenarios with different parameters and perform the simulations (see Table I). In first scenario, we took 100 nodes in $100 \mathrm{~m} \times 100 \mathrm{~m}$ network with initial energy $0.5 j$, which is same as original SEP routing protocol. In second scenario, we took 150 nodes in $200 m \times 200 m$ network with initial energy $1 j$. In third scenario, we took the heterogeneity on next level with $1.5 j$ initial energy while the number of nodes are 200 and the network dimensions are $250 \mathrm{~m} \times 250 \mathrm{~m}$.

\section{TABLE I. PARAMETERS USED IN Simulation}

\begin{tabular}{|l|c|}
\hline Parameter & Value \\
\hline Scenario & $\mathbf{1}$ \\
\hline Number of Nodes & 100 \\
Network Dimension & $100 \mathrm{~m} \times 100 \mathrm{~m}$ \\
Initial Energy & $0.5 \mathrm{j}$ \\
\hline Scenario & $\mathbf{2}$ \\
\hline Number of Nodes & 150 \\
\hline Network Dimension & $200 \mathrm{~m} \times 200 \mathrm{~m}$ \\
Initial Energy & $1 \mathrm{j}$ \\
\hline Scenario & $\mathbf{3}$ \\
\hline Number of Nodes & 200 \\
Network Dimension & $250 \mathrm{~m} \times 250 \mathrm{~m}$ \\
Initial Energy & $1.5 \mathrm{j}$ \\
\hline Scenario & $\mathbf{4}$ \\
\hline Number of Nodes & 250 \\
Network Dimension & $300 \mathrm{~m} \times 300 \mathrm{~m}$ \\
Initial Energy & $2 \mathrm{j}$ \\
\hline Number of rounds & 5000 \\
Percentage of being Cluster Head & .1 \\
$E_{D A}$ Energy cost & $50 \mathrm{pj} / \mathrm{bit} \mathrm{j}$ \\
packet size & $4000 \mathrm{bit}$ \\
$E_{T X}$ & $50 \mathrm{jj} / \mathrm{bit}$ \\
$E_{R X}$ & $50 \mathrm{nj} / \mathrm{bit}$ \\
$E_{f s}$ & $100 \mathrm{pj} / \mathrm{bit} / \mathrm{m}^{2} / \mathrm{m}^{2}$ \\
$E_{a m p}$ & \\
\hline & \\
\hline
\end{tabular}

Last, but not least we deployed 250 nodes in the field of $300 \mathrm{~m} \times 300 \mathrm{~m}$ with the initial energy of $2 j$. In order to further prove the scalability of SEP routing protocol, we took the simulation on higher level of heterogeneity with 


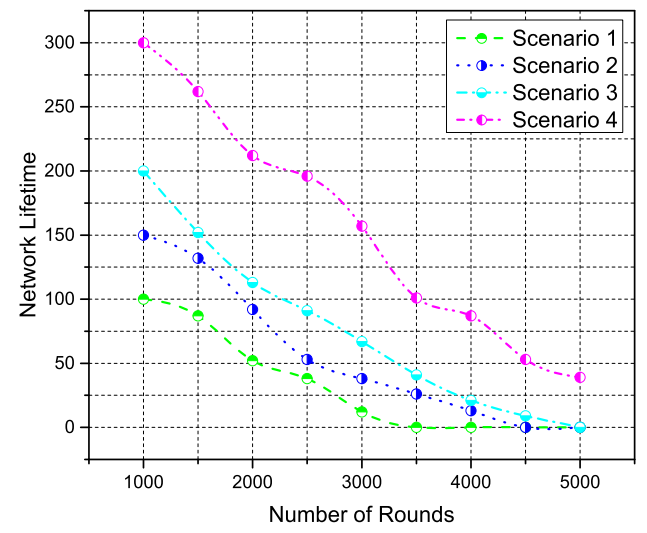

Fig. 6. Network lifetime.

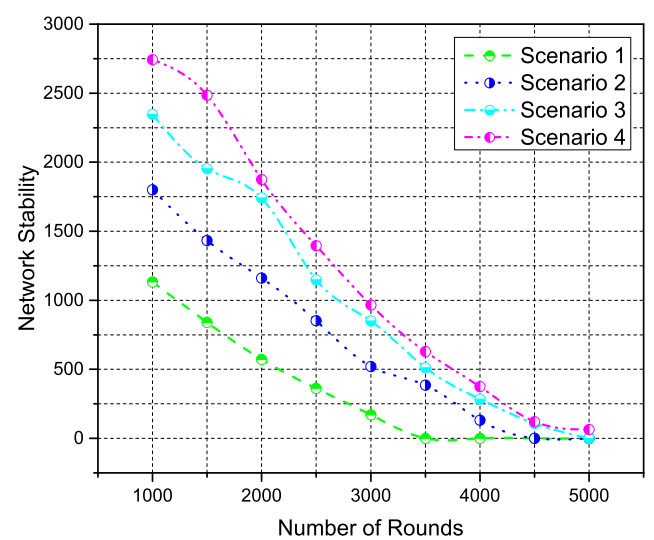

Fig. 7. Network stability.

more more sensor nodes in the larger network. Simulation results prove that SEP routing protocol is reliable in large scale network. Detailed simulation parameters are given in Simulation Parameters table.

Fig. 6 shows the network lifetime of SEP routing protocol in four different scenarios. It can be seen that SEP perform more better in large network. Figure shows that in larger network, the network lifetime is $110 \%$ increased from the standard SEP routing protocol mentioned in scenario 1.

Network stability is shown in Fig. 7. It can be seen that stability of standard SEP routing protocol is 1110 rounds. Scenario 2 shows the second level of heterogeneity which is $90 \%$ higher than original routing protocol with the stability of 1700 rounds. Scenario 3 showing the results in similar fashion, with the stability of 2341 rounds which is $150 \%$ improved than standard SEP routing protocol. While in scenario 4, network is stable till 2753 rounds. Figure proves that SEP routing protocol is stable and reliable for large scale network.

As we deploy the network in bigger environment, sensor nodes consumes more energy. Standard SEP routing protocol

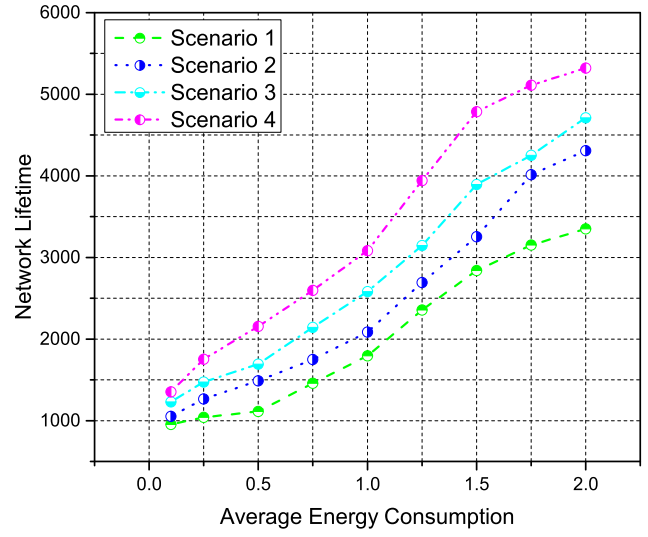

Fig. 8. Average energy consumption.

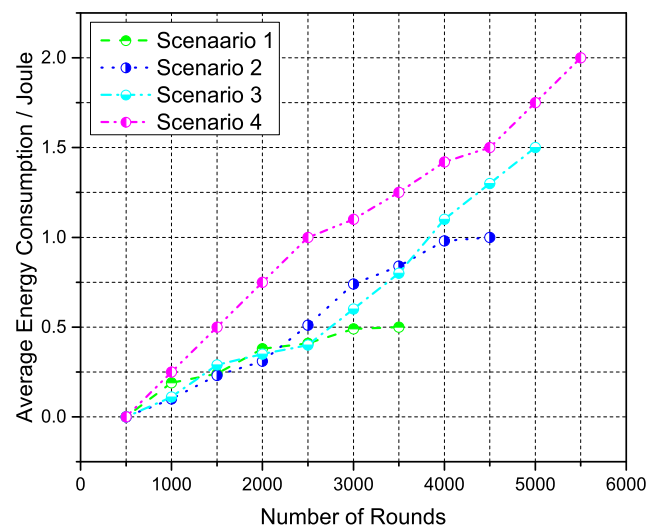

Fig. 9. Average energy consumption per round.

consumes minimum energy because of small size of network dimensions. Fig. 8 shows the average energy consumption on different level heterogeneity. Proposed scenarios consumes more energy because of large network size and higher number of nodes.

As we have mentioned in the simulation parameters, we took the four different scenarios to prove the scalability. It can be seen clearly that SEP protocol enhances its network lifetime in large scale. But as the network size increases, sensor nodes consumes more energy because of communication gap between nodes and CHs. Fig. 4 shows the energy consumption with respect to number of rounds. If we focus on scenario 4 in Fig. 9 , it can be seen clearly that there is huge difference between standard SEP protocol and scalable SEP protocol. In scalable network, SEP improves network lifetime but consumes more energy which is the only drawback of SEP routing protocol in large scale networks.

Efficient data packet delivery is essential for any routing protocol, Fig. 10 shows the reliability of packet delivery by calculating the delay in network. Figure proves that SEP rout- 


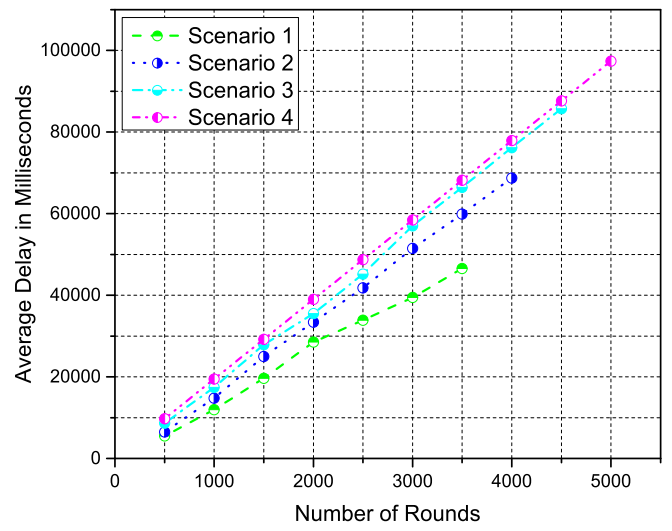

Fig. 10. Network delay.

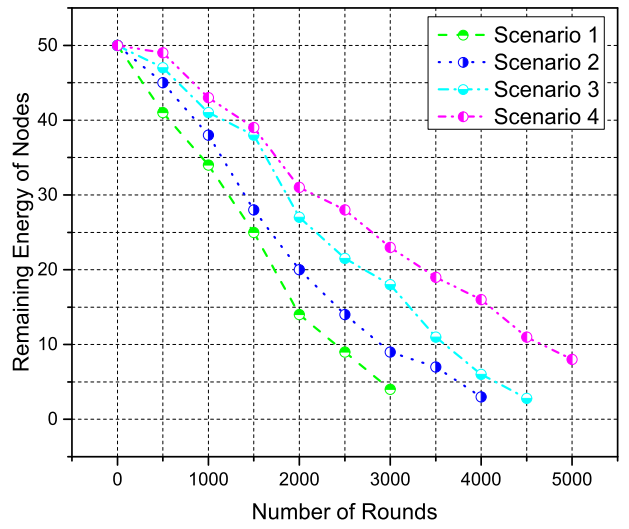

Fig. 11. Remaining energy of sensor nodes.

ing protocol minimize the delay in data packet transmission as well as data packet reception. Figure shows the potential of SEP routing protocol that it enhances the packet delivery ratio by minimizing the average delay in network.

Residual energy of sensor nodes is calculated after every round. As we describe earlier that SEP routing protocol use epoch to select a new $\mathrm{CH}$ after each round to balance the load of network. This technique of $\mathrm{CH}$ selection is truly based on residual energy of sensor nodes. Nodes with the higher energy level have more probability to become $\mathrm{CH}$. Fig. 11 shows the remaining energy of sensor nodes with respect to nodes. It can be seen clearly that there is no big difference in remaining energy for standard SEP and in large scale. Protocol distribute the network load among all sensor nodes which makes the reliability in large scale network. Small variation in figure is because of larger network and large number of sensor nodes.

After simulating SEP protocol in above mentioned 4 scenarios, we took the heterogeneity from $0.5-5 j$ for better comparison. Fig. 12 shows the impact of different heterogeneity levels with respect to different number of sensor nodes

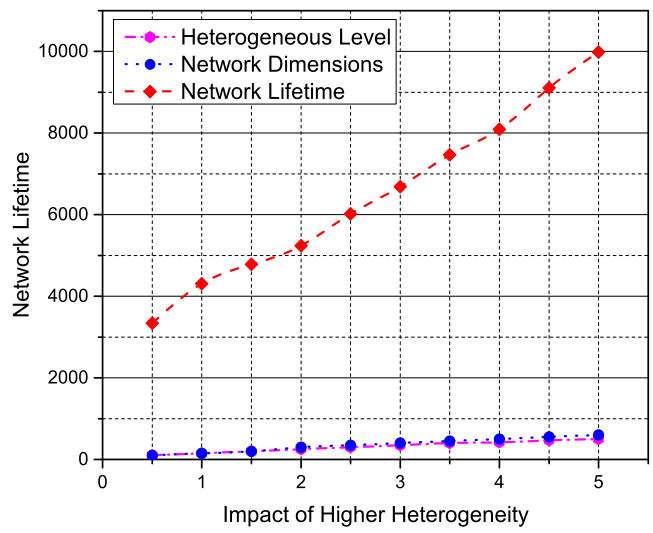

Fig. 12. Impact of higher heterogeneity level.

and different level of network dimensions. Figure shows the stability and reliability of SEP routing protocol that it works more stable in large scale network. It can be seen that network lifetime also increases as we increase the heterogeneity level. Figure shows the potentials of routing protocol and it also proves the objective of this paper.

\section{CONCLUSION}

We propose the scalability of SEP routing protocol with the fourth level of heterogeneity for large scale traffic monitoring. The selection of cluster-heads are independent and elected efficiently through random $\mathrm{CHs}$ selection technique. Two types of nodes are deployed in the network with different heterogeneous level as similar in the SEP routing protocol. In order to prove the reliability in large scale network, we deployed four different types of heterogeneous nodes in the simulation experiments. Results shows the behavior of SEP routing routing protocol in large scale network. After proving the reliability in four different network dimensions, we further check the impact of heterogeneity in huge network. Where, the heterogeneity is extended to the 10th level and network dimension is extended to $500 \%$ greater than the standard SEP routing protocol. Experimented results shows that SEP routing protocol works more better in large scale network and enhances the lifetime with more stable network.

\section{ACKNOWLEDGMENT}

We are thankful to the authors of SEP routing protocol for providing code and results online.

\section{STATEMENT OF CONFLICT}

Authors of this paper: Muhammad Asad, Arsalan Ali Shaikh, Soomro Pir Dino, Muhammad Aslam and Yao Nianmin declares that there is no conflict of interest regarding the publication of this research article entitled "Lifetime Maximization on Scalable Stable Election Protocol for Large Scale Traffic Engineering". 


\section{REFERENCES}

[1] Kafi, Mohamed Amine, Jalel Ben Othman, and Nadjib Badache. "A Survey on Reliability Protocols in Wireless Sensor Networks." ACM Computing Surveys (CSUR) 50.2 (2017): 31.

[2] A. Roshini and H. Anandakumar, Hierarchical Cost Effective LEACH for Heterogeneous Wireless Sensor Networks, in Advanced Computing and Communication Systems, 2015 International Conference on, Jan 2015, pp. 17.

[3] M. Pramanick, P. Basak, C. Chowdhury, and S. Neogy, Analysis of energy efficient wireless sensor networks routing schemes, in Fourth International Conference of Emerging Applications of Information Technology (EAIT), 2014, Dec 2014, pp. 379384.

[4] Rehan, Waqas, et al. "A comprehensive survey on multichannel routing in wireless sensor networks." Journal of Network and Computer Applications 95 (2017): 1-25.

[5] Wang, J.; Zhang, Z.; Xia, F.; Yuan, W.; Lee, S. An energy efficient stable election-based routing algorithm for wireless sensor networks. Sensors 2013, 13, 1430114320.

[6] Wang, Jin, et al. "Analysis of energy consumption in direct transmission and multi-hop transmission for wireless sensor networks." Signal-Image Technologies and Internet-Based System, 2007. SITIS'07. Third International IEEE Conference on. IEEE, 2007.

[7] Hammoudeh, M.; Newman, R. Adaptive routing in wireless sensor networks: QoS optimisation for enhanced application performance. Information Fusion 2015, 22, 315.

[8] M Shamsan Saleh, A.; Mohd Ali, B.; A Rasid, M.F.; Ismail, A. A self-optimizing scheme for energy balanced routing in wireless sensor networks using sensorant. Sensors 2012, 12, 1130711333.

[9] Heinzelman, Wendi Rabiner, Anantha Chandrakasan, and Hari Balakrishnan. "Energy-efficient communication protocol for wireless microsensor networks." System sciences, 2000. Proceedings of the 33rd annual Hawaii international conference on. IEEE, 2000.

[10] Smaragdakis, Georgios, Ibrahim Matta, and Azer Bestavros. SEP: A stable election protocol for clustered heterogeneous wireless sensor networks. Boston University Computer Science Department, 2004.

[11] Al-Anbagi, Irfan, Melike Erol-Kantarci, and Hussein T. Mouftah. "A survey on cross-layer quality-of-service approaches in WSNs for delay and reliability-aware applications.” IEEE Communications Surveys \& Tutorials 18.1 (2016): 525-552.

[12] Chang, Feng-Cheng, and Hsiang-Cheh Huang. "A survey on intelligent sensor network and its applications." Journal of Network Intelligence 1.1 (2016): 1-15.

[13] Rostami, Ali Shokouhi, et al. "Survey on clustering in heterogeneous and homogeneous wireless sensor networks." The Journal of Supercomputing (2017): 1-47.
[14] Alwajeeh, Taha, et al. "Efficient method for associating radio propagation models with spatial partitioning for smart city applications." Proceedings of the International Conference on Internet of things and Cloud Computing. ACM, 2016.

[15] Fujii, Shohei, et al. "Optimal cluster head selection and rotation of cognitive wireless sensor networks for simultaneous data gathering." Information Networking (ICOIN), 2017 International Conference on. IEEE, 2017.

\section{Authors' Profile}

Muhammad Asad received his B.S. degree in Telecommunication and Networks from COMSATS Institute of Information Technology, WAH CANTT, Pakistan in 2014. Now he is pursuing his masters degree in School of Computer Science and Technology, Dalian University of Technology, China. His main research interests include Wireless Sensor Networks, Internet of Things, and information security.

Arsalan Ali Shaikh was born in (1990). He received his Bachelor Degree in Software Engineering from the University of Sindh Jamshoro Pakistan in (2012). After that he served as a Software Developer in private software organization in 2013. Currently, he is doing Master Degree in Computer Science and application technology in Dalian University of Technology, P.R. China. His research interest area include Big Data, Cloud Computing and Computer Networks.

Soomro Pir Dino was born in (1989); He received his Bachelor Degree in Bachelor of Science Information Technology BSIT (Hons.) from the Sindh Agriculture University (SAU) Tandojam, Pakistan in 2013. Currently he is doing Masters Degree (MS) in Computer Science and Technology in Dalian University of Technology (DUT), Dalian, P. R. China. His research Interest is Data Mining, Machine Learning and Interconnected Networks and Topological Structure.

Muhammad Aslam received the B.S degree in Telecommunication System and the M.S degree in Electrical Engineering from BZU Multan and COMSATS Institute of Information Technology Islamabad in 2010, 2012, respectively. He was Lecturer at COMSATS Institute of Information Technology, Wah Cantt. His major research interests are Energy optimization in WSNs, WBANs, and UWSNs. Currently he is $\mathrm{PhD}$ scholar at School of Computer Science and Technology, Dalian University of Technology, Dalian, China.

Yao Nianmin received the B.E., M.S. and Ph.D degree from Jilin University. He is currently a professor in Dalian University of Technology. He has been a visiting scholar at University of Connecticut. His Primary research interests include Wireless Sensor Networks and Wireless Network Security. 